\title{
Studies on Dynamics of Driven Bilaterally Coupled Gunn Oscillator
}

\author{
J. Chakravorty ${ }^{1}$, S. Sarkar ${ }^{2}$ and B. C. Sarkar ${ }^{1 *}$ \\ 'Department of Physics, The University of Burdwan, Golapbag, Burdwan-713104, West Bengal, \\ India; bcsarkar_phy@yahoo.co.in \\ 2Department of Electronics, Burdwan Raj College, Burdwan-713104, West Bengal, India
}

\begin{abstract}
The dynamics of bilaterally coupled Gunn oscillator driven by external periodic signal has been examined through numerical simulation and experimental observation. The coupling factors between the two oscillators in either path as well as the strength and frequency of the external periodic driving signals are key parameters to control the dynamics of Driven Bilaterally Coupled Gunn Oscillator (DBCGO) system. The numerical study explores the possibility of generation of controllable chaotic signals in DBCGO system. The present work also considers this method through a hardware experiment. For different set of coupling factors, driving signal strength and frequencies, chaotic oscillations are observed. Therefore, the DBCGO system with suitable couplings and driving signal conditions can be considered as a potential source of chaotic signals in microwave frequency range.
\end{abstract}

Keywords: Coupled System, Driven Oscillator, Gunn Diode, Microwave Chao

\section{Introduction}

Recently, coupled oscillator systems have drawn attention of the researchers of different fields like theoretical physics, engineering science, biological science, chemical science and even economics and social sciences ${ }^{1,2}$. Different models of such coupled oscillators have been suggested by the researchers to study the dynamics of such systems $s^{3,4}$. In the area of electronics and communication technology, coupled oscillator systems find applications in stable signal generation, power combining, synchronous amplifications and so on ${ }^{5,6}$. At microwave frequency range, coupled oscillator systems are considered for power combining, spatial beam forming and phase shifting ${ }^{7,8}$. Chaos in such coupled oscillator array has also been discussed ${ }^{9}$. Recently, the dynamics and chaos in Bilaterally Coupled Gunn Oscillators (BCGO) has been reported ${ }^{10}$. It has been shown that, depending on free running frequencies of each oscillator and Coupling Factors (CF) between them, chaotic oscillations can be obtained.
In this paper, the dynamics of a BCGO system, subjected to an external periodic driving signal has been studied. The BCGO has been set to operate in the synchronized mode of oscillations and an external signal is applied to one of the oscillators to examine the effect of forcing. The frequency of the external driving signal is varied keeping its power fixed at value and output spectra of the system are observed. The aim of the paper is to address the following problems:

(i) To find the effect of the external driving signal on the Synchronization Band (SB) of the BCGO system.

(ii) To explore through numerical study the possibility of generation of controllable chaotic signals in microwave (MW) frequency range using a driven BCGO system.

(iii) To experimentally study the dynamics of driven BCGO system in the MW frequency domain.

The paper has been organized in the following way. Section 2 describes the Driven BCGO (DBCGO) and

${ }^{*}$ Author for correspondence 
obtains its suitable equivalent circuit theoretic model. A differential equation based on the equivalent circuit describing the dynamics of such GO is proposed. In Section 3, the system equations are numerically solved. From numerical studies, the tuning characteristic of individual GO, the SB of the BCGO and some chaotic oscillation states of DBCGO are presented. The experimental study on the dynamics of DBCGO system has been reported in Section 4. It describes the hardware realization of the proposed system, method of experimental study, results and discussions. Section 5 presents some concluding remarks on these studies.

\section{Equivalent Model of Driven Bilaterally Coupled Gunn Oscillators}

The simplified functional block diagram of the DBCGO is shown in Figure 1. Here two Gunn Oscillators (GO) (indicated by GO-1 and GO-2) coupled to each other by circulators. Also CRs are required to apply an external signal to a GO and to take output signal from a GO. For bidirectional coupling two CRs are required. The respective GOs are connected to the port-2 of concerned CRs while the coupling signals are applied at port- 1 and output is taken out from port- 3 of each CR. For controlling the coupling in both directions, two attenuators (ATN) are used. The external driving signal has been applied to one of the Gunn oscillators (GO-1 in the present work) and the output has been observed.

Figure 2 shows an equivalent circuit theoretic model of the DBCGO system under study. Here, a single GO is modeled as a series combination of the device impedance and the cavity impedance ${ }^{10}$. In the model, the diode in the active region is replaced by a voltage dependent negative resistor $R_{D}$ and capacitor $C_{S} \cdot v_{R}$ and $v_{C}$ are the voltage drops across them. The waveguide cavity formed

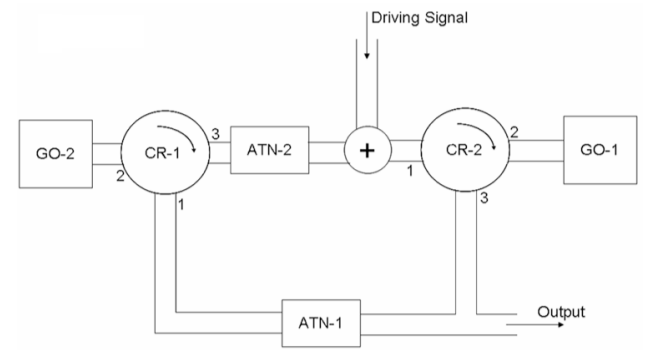

Figure 1. Simplified functional structure of DBCGO.

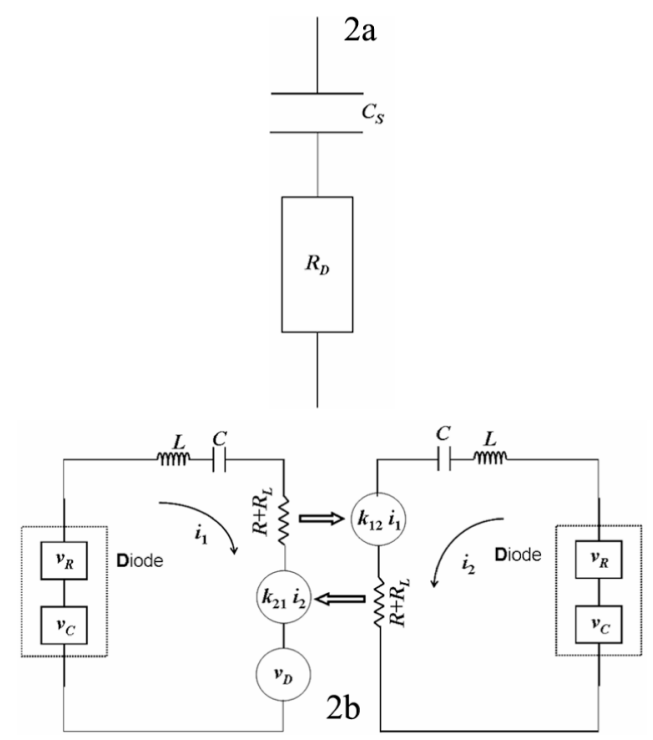

Figure 2. (a) Series equivalent circuit of unpackaged Gunn diode and (b) Equivalent circuit of DBCGO.

by a rectangular waveguide section terminated with a movable short circuit is represented by a series resonant circuit comprising of a resistor $R$, inductor $L$ and capacitor $C$. Since $R_{D}$ and $C_{S}$ are both nonlinear in nature, the voltage drops $v_{R}$ and $v_{C}$ would be nonlinear functions of the instantaneous current $i=\frac{d q}{d t}$ and instantaneous charge $q(t)$ respectively. Considering up to the cubic nonlinearity term and remembering that the device offers negative resistance, $v_{R}$ and $v_{C}$ can be expressed as follows:

$$
\begin{aligned}
& v_{R}=-\beta_{1} i+\beta_{3} i^{3} \\
& v_{C}=\alpha_{1} q+\alpha_{3} q^{3}
\end{aligned}
$$

Here $\beta_{1}, \beta_{3}, \alpha_{1}$, and $\alpha_{3}$ are the device parameters and their magnitude would depend on the $\mathrm{dc}$ bias point. Among them $\beta_{1}$ and $\beta_{3}$ represents the linear and nonlinear relation between voltage drop across the nonlinear resistor of the device and current through it. The magnitude of $\beta 1$ and $\beta 3$ are dependent on the applied bias voltage. Beyond threshold level of bias voltage, $\beta_{1}$ is negative. The parameters $\alpha_{1}$ and $\alpha_{3}$ are related with the linear and nonlinear part of the diode capacitance. Higher order terms beyond cubic ones are ignored because as far as periodic oscillation is concerned, neglect of such higher powers results in no remarkable change in output ${ }^{11}$. The second order term is also neglected, as it would contribute only to $\mathrm{dc}$ as well as a frequency component of second harmonic of the free running frequency of oscillation and it would be filtered out by the resonant circuit. The differential equation of a single GO then can be obtained as ${ }^{10,12}$ : 


$$
\frac{d^{2} q}{d \tau^{2}}=a q-b q^{3}+c \frac{d q}{d \tau}-d\left(\frac{d q}{d \tau}\right)^{3}
$$

where, $\tau=\omega_{R} t$ is the normalized time. The coefficients $a, b, c, d$ are dependent on both bias voltage through device parameters $\beta_{1}, \beta_{3}, \alpha_{1}$, and $\alpha_{3}$ as well as waveguide cavity parameters $R, L$ and $C$ along with external load $\mathrm{RL}$. When two such GOs are bidirectionally coupled, the effect of coupling can be circuit theoretically modeled by including two voltage sources as indicated in Figure 2. These voltage sources in the equivalent circuit is to be taken as proportional to the difference of currents flowing through the two oscillators. The external driving signal is applied to GO-1 and represented as another voltage source in the equivalent circuit for GO-1. Hence following the system equations for GO- 1 and GO-2 of DBCGO can be written as follows:

$$
\begin{aligned}
\frac{d^{2} q_{1}}{d \tau^{2}}= & a_{1} q_{1}-b_{1} q_{1}^{3}+c_{1}\left(\frac{d q_{1}}{d \tau}\right)-d_{1}\left(\frac{d q_{1}}{d \tau}\right)^{3}+ \\
& k_{21}\left(\frac{d q_{2}}{d \tau}-\frac{d q_{1}}{d \tau}\right)+\varepsilon \cos \left(\Omega_{D} \tau\right) \\
\frac{d^{2} q_{2}}{d \tau^{2}}= & a_{2} q_{2}-b_{2} q_{2}^{3}+c_{2}\left(\frac{d q_{2}}{d \tau}\right)- \\
& d_{2}\left(\frac{d q_{2}}{d \tau}\right)^{3}+k_{12}\left(\frac{d q_{1}}{d \tau}-\frac{d q_{2}}{d \tau}\right)
\end{aligned}
$$

Here $k^{21}\left(k^{12}\right)$ is the coupling factor (CF) in the path GO-2 to GO-1 (GO-1 to GO-2). ( $\left.\frac{d q_{1}}{d \tau}\right)$ and $\left(\frac{d q_{2}}{d \tau}\right)$ represent the instantaneous circulating current through the equivalent circuit of GO-1 and GO-2 respectively. $\varepsilon$ and $\Omega_{D}$ are the amplitude and normalized frequency of the external driving signal.

In the present work, before applying the external driving signal, GO-1 and GO-2 are adjusted to oscillate in the synchronized state and the common frequency of synchronized oscillation is given ${ }^{10}$

$$
\Omega_{0}=\left[\frac{k_{12} A_{1}^{2} \Omega_{1}^{2}+k_{21} A_{2}^{2} \Omega_{2}^{2}}{k_{12} A_{1}^{2}+k_{21} A_{2}^{2}}\right]^{\frac{1}{2}}
$$

Then the external signal has been added and depending on its amplitude and frequency, quasiperiodic, chaotic and synchronized state of oscillations could be observed.

\section{Numerical Results}

The dynamics of DBCGO has been studied solving the system equations numerically. For this, the system equations (4) and (5) are decomposed into two pairs of first order differential equations as below:

$$
\begin{aligned}
& \frac{d q_{1}}{d \tau}=p_{1} \\
& \frac{d q_{2}}{d \tau}=p_{2} \\
& \frac{d p_{1}}{d \tau}=a_{1} q_{1}-b_{1} q_{1}^{3}+c_{1} p_{1}-d_{1} p_{1}^{3}+k_{21} p_{2}+\varepsilon \cos \left(\Omega_{D} \tau\right) \\
& \frac{d p_{2}}{d \tau}=a_{2} q_{2}-b_{2} q_{2}^{3}+c_{2} p_{2}-d_{2} p_{2}^{3}+k_{12} p_{1}
\end{aligned}
$$

The equations (7) to (10) have been solved using fourth order Runge-Kutta (RK) technique. The output of the oscillator is studied by noting the time variation of $q^{1,2}$, drawing the phase plane plot of $q^{1,2}$ and $p^{1,2}$ and examining the frequency components present in the time varying signal. This frequency domain response is obtained by Discrete Fourier Transform (DFT) of time samples. In order to have the steady state response of the system, a sufficient number of samples of state variables of $q^{1,2}$ and $p^{1,2}$ from initial time have not taken into account. The following observations have been made from the numerical study:

- In the free running operation of GO, obtained with $k^{21}=k^{12}=0$ and $\varepsilon=0$, one can have four parameters $a, b, c$ and $d$ for numerical tuning of individual GOs. Among them, $a$ and $b$ are related to the capacitive part of the diode as well as cavity. An oscillation would be obtained when the bias voltage of the device is beyond threshold level i.e. the device operates in negative resistance region. In the equations of free running GO, this condition is applied through the parameter $c$. The parameter $d$ is related to the device parameter $\beta^{3}$. It has the responsibility to check the growth of oscillation amplitude beyond a certain limit. Therefore the value of parameter $d$ is to be taken as less than $c$ for GO. We have chosen the values of $a, c$ and $d$ are taken as 1.0, 0.05 and 0.015 respectively and $b$ has been varied to tune the frequency of oscillation. The numerically obtained variations in oscillation frequency with the parameter $b$ at two different bias points (i.e. for two different 
values of $c$ ) are shown in Figure 3. Likewise, numerically obtained variations in oscillation frequency with the parameter $c$ for two different mechanical tuning conditions (i.e. for two different values of $b$ ) are shown in Figure 4.

- The common frequency of oscillation of the BCGO has been obtained by adjusting the values of the device and cavity parameters and CFs. It has been observed that synchronization band (SB) of the system increases with decrease in $k^{12}$ i.e. CF in reverse path and increases with $k^{21}$ i.e. CF in forward path (Table 1).

- Figures 5 and 6 show the quasiperiodic output (spectrum and phase-plane plot) of DBCGO system at two different conditions. The frequency spectrum consists of a number of discrete frequency components and phase plane plot show a band type nature which are the indication of such quasiperiodic state of operation. The quasiperiodic state of oscillation can occur when the frequencies are incommensurate.

- Figures 7 and 8 show the simulated output spectrum of DBCGO system for two different driving signal powers along with the phase-plane plots of GO output variables at two different conditions. Occurrence

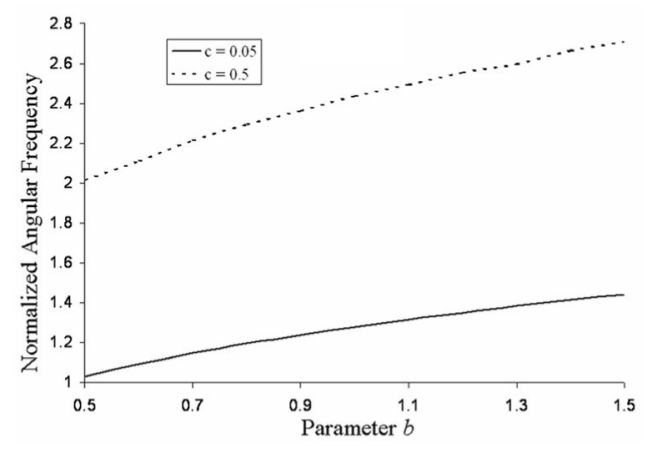

Figure 3. Simulated variations of normalized angular frequency of individual GO with parameter.

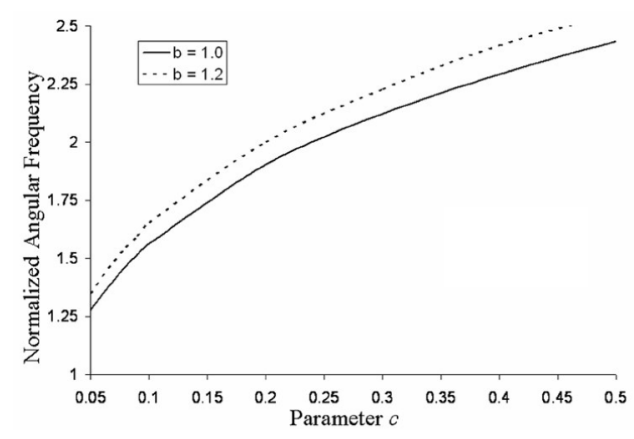

Figure 4. Simulated variations of normalized angular frequency of individual GO with parameter $c$. of chaotic oscillation is evident from the broad output spectrum and the double-scroll type phase plane plot showing strange attractors.

\section{Experimental Results}

\subsection{Experimental Arrangement}

To study the DBCGO system experimentally, a hardware arrangement has been obtained according to the

Table 1. Variation of simulated SB of DBCGO with a set of CFs when driving signal is not present

\begin{tabular}{ccc}
\hline \multicolumn{2}{c}{ Coupling Factor } & Simulated SB (Normalized) \\
\cline { 1 - 2 } $\boldsymbol{k}^{21}$ & $\boldsymbol{k}^{12}$ & \\
\hline 0.25 & 0.25 & 0.0023 \\
0.25 & 0.10 & 0.203 \\
0.25 & 0.005 & 0.393 \\
0.15 & 0.15 & 0.02 \\
0.20 & 0.15 & 0.186 \\
0.25 & 0.15 & 0.305 \\
0.30 & 0.15 & 0.388 \\
\hline
\end{tabular}
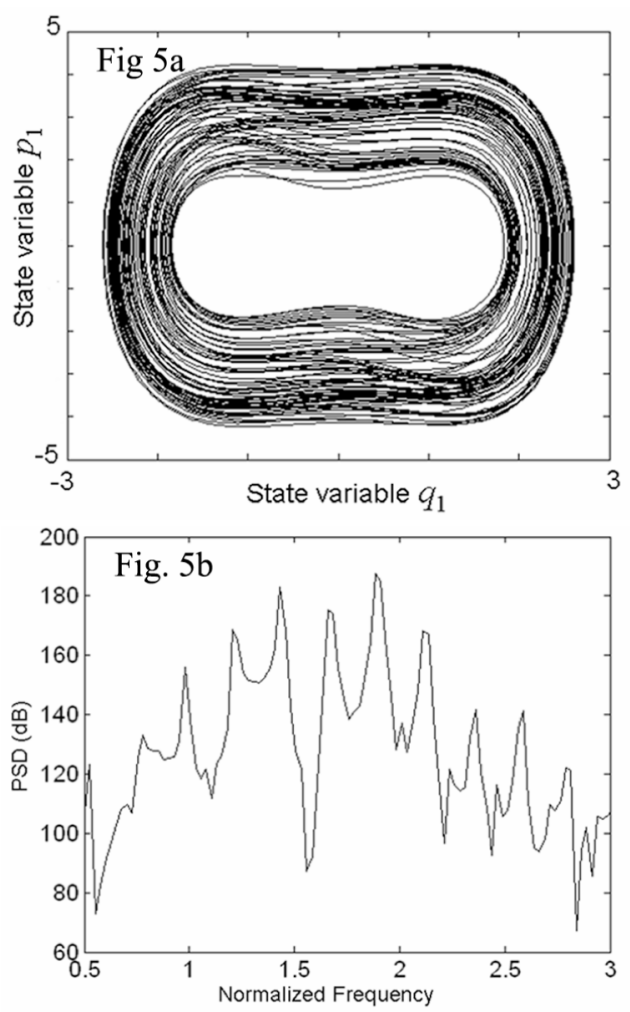

Figure 5. Simulated output of DBCGO: $k^{21}=0.25, k^{12}=$ $0.10, \Omega^{0}=1.4025, \Omega^{D}=1.10, \varepsilon=0.50$ : (a) phase-plane plot, (b) frequency spectrum. 

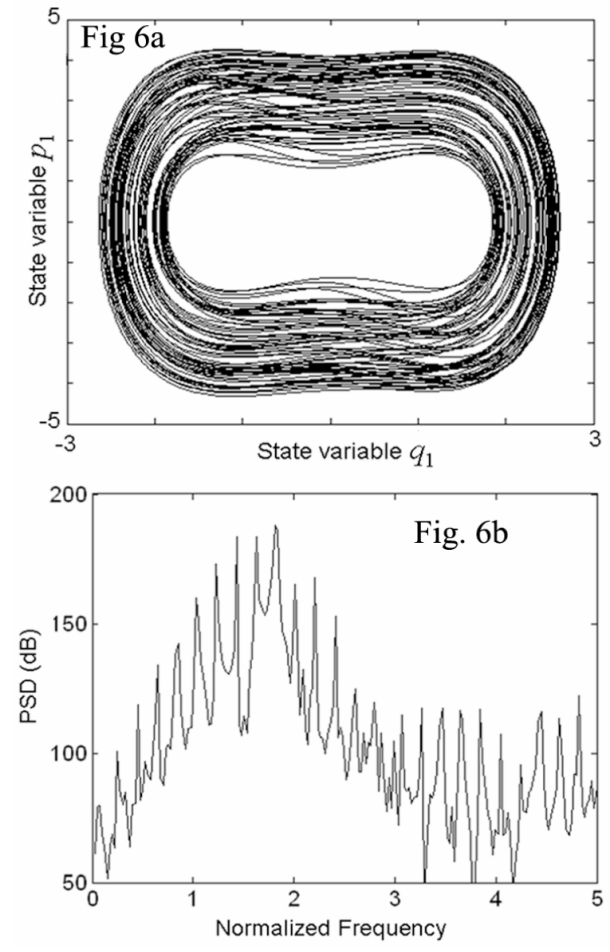

Figure 6. Simulated output of DBCGO: $k^{21}=0.25, k^{12}=$ $0.10, \Omega^{0}=1.4025, \Omega^{D}=1.21, \varepsilon=0.65$ : (a) phase-plane plot, (b) frequency spectrum.
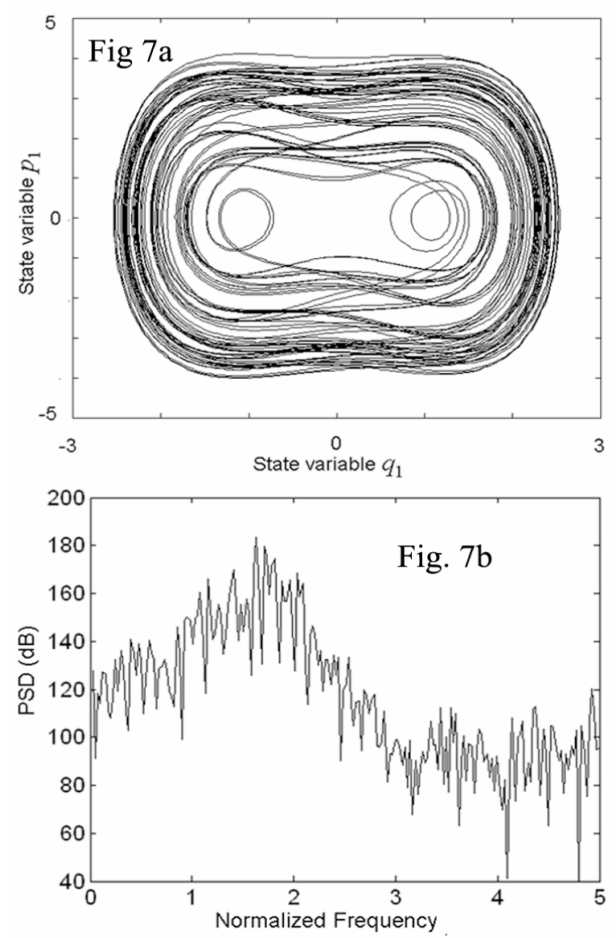

Figure 7. Simulated output of DBCGO: $k^{21}=0.25, k^{12}=$ 0.10, $\Omega^{0}=1.4025, \Omega^{D}=1.16, \varepsilon=0.50$ : (a) phase-plane plot, (b) frequency spectrum.
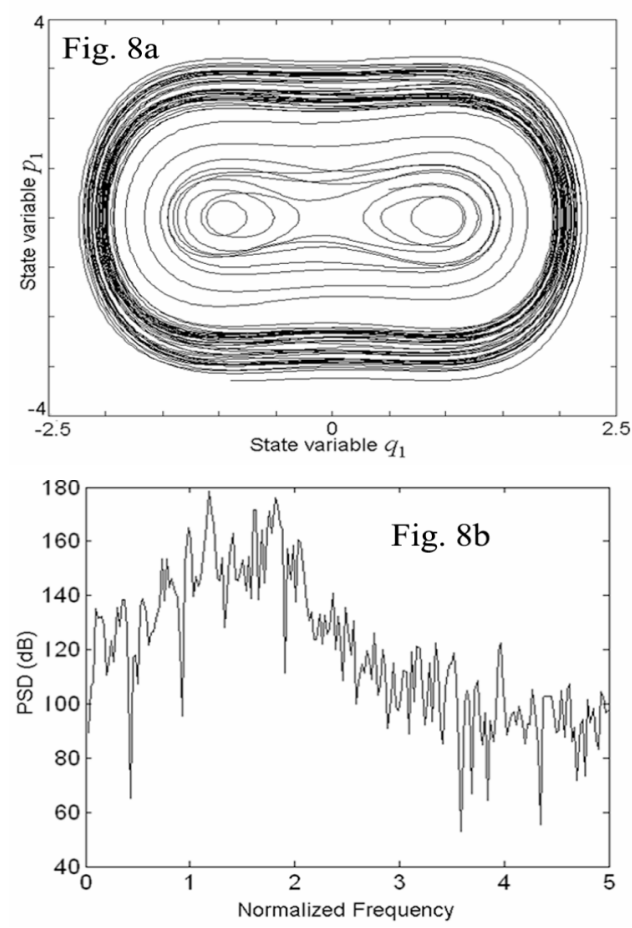

Figure 8. Simulated output of DBCGO: $k^{21}=0.25, k^{12}=$ $0.10, \Omega^{0}=1.4025, \Omega^{D}=1.21, \varepsilon=0.65$ : (a) phase-plane plot, (b) frequency spectrum.

functional-block diagram as shown in Figure 1. The major active and passive components of the experimental set-up are Gunn oscillators, circulators, magic tee, attenuators, Spectrum analyzer and microwave signal source. The free running frequency of GO-1 and GO-2 are initially adjusted to $10 \mathrm{GHz}$ with output power of about $20.2 \mathrm{~mW}$ and $22.4 \mathrm{~mW}$ respectively. The frequencies of the respective oscillators have been mechanically controlled with the help of micrometer screws attached with the waveguide structure of the respective GOs. The CFs between the oscillators in two paths are selected with the two adjustable attenuators (ATN-1 and ATN-2). The CFs are determined by measuring the ratios of output powerto-input power in each path by adjusting the respective micrometer screws of variable attenuators. The external driving signal (frequency $f^{d}$ and power $P^{d}$ ) is taken from the microwave signal source and applied to GO-1 of the coupled system. The output spectra are recorded with a spectrum analyzer to study the dynamics of the system.

\subsection{Experimental Observations}

In the first part of the experiment, the effect of two CFs on the SB of a particular GO (GO-1 in the present case) 
has been studied. It has been observed that, the SB of GO-1 (i) increases due to increase in $k^{21}$ for fixed $k^{12}$ and (ii) decreases due to increase in $k^{12}$ for fixed $k^{21}$. Figure 9 shows the measured SB of GO-1 for different set of CFs.

Then, for a set of CFs, the common frequency of oscillation $\left(f^{0}\right)$ has been obtained and SBs are measured for different driving signal strengths $\left(P^{d}\right)$. The measured results are summarized in Table-2. From the result following observations could be made:

(i) A minimum $P^{d}$ is required to obtain a $\mathrm{SB}$ of a driven GO when it is already mutually synchronized with another GO.

(ii) For fixed values of $k^{21}$ and $k^{12}$, the $\mathrm{SB}$ of GO-1 increases with $P^{d}$.

(iii) For fixed values of $P^{d}$, the SB of GO-1 increases with increase in $k^{12}$ while mutual SB reduces.

In the second part of experiment, both GO-1 and GO-2 are made to oscillate at $f^{0}=10 \mathrm{GHz}$ for different sets of values of $k^{21}$ and $k^{12}$ and external driving signal are applied. The $f^{d}$ and $P^{d}$ are the two parameters which are used to study the effect of external driving signal on corresponding GO dynamics. Therefore, for each combination of CFs, $f^{d}$ has been varied around $f^{0}$ for some

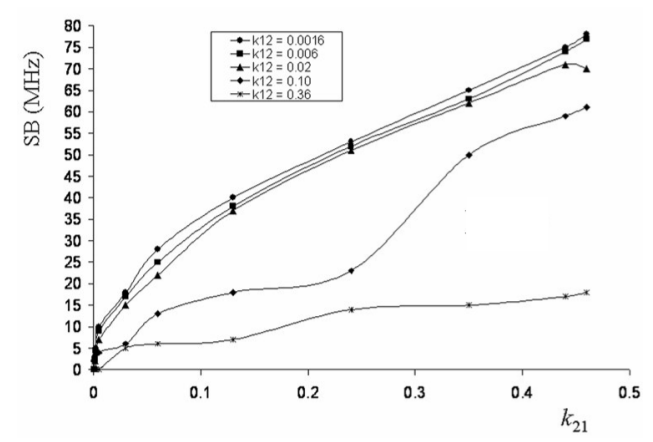

Figure 9. Measured SB of GO-1 for different set of CFs. fixed $P^{d}$. When $f^{d}$ is far from $f^{0}$ and at the lower side, there are some windows in the frequency span of the driving signal where a number of complex behaviors like quasi periodic nature of oscillation and random variation in its amplitude showing broad band continuous spectrum are observed. The quasi periodic state occurs when the ratio between $f^{0}$ and $f^{d}$ is an irrational number. However, adjusting the strength and the frequency of the driving signal, apparent random behavior can be observed in the GO dynamics. This suggests that in this condition of chaotic state of oscillation can be obtained from the DBCGO. With further increase in $f^{d}$, quasi periodic state is again observed in 'out of lock' condition. However, only one cases of occurrence of chaotic state has been recorded at higher side of SB. From these results, it can be stated that depending on $P^{d}$, the following transitions are possible when $f^{t}$ approaches $f^{0}:$ (i) quasiperiodic $\rightarrow$ chaotic $\rightarrow$ quasiperiodic $\rightarrow$ synchronized, (ii) quasiperiodic $\rightarrow$ chaotic $\rightarrow$ synchronized, (iii) quasiperiodic $\rightarrow$ chaotic, (iv) quasiperiodic $\rightarrow$ chaotic $\rightarrow$ quasiperiodic $\rightarrow$ chaotic $\rightarrow$ synchronized. In the next part of the experiment, the free running frequency of GO-1 has been set at $10 \mathrm{GHz}$ and frequency of GO-2 has been adjusted so that $f^{0}$ attains a value near one extreme end of SB. Then the effect of external driving signal has been studied for different values of CFs as well as $f^{d}$ and $P^{d}$. In these situations also quasiperiodic, chaotic and synchronized state of oscillations have been observed. Unlike the previous case, chaotic responses are observed at the both side of SB. Some of the observed output spectra of the DBCGO for above two situations are presented in Figure 10 and 11 respectively.

\section{Conclusions}

In this paper, the dynamics of X-band DBCGO has been studied. A suitable circuit theoretic model of such DBCGO system is described at the outset. Based on the

Table 2. Measured SB of GO-1 of DBCGO for different coupling and driving signal condition

\begin{tabular}{|c|c|c|c|c|c|c|}
\hline \multicolumn{2}{|c|}{ Coupling Factor } & \multirow{3}{*}{$\begin{array}{c}\text { SB }(\mathrm{MHz}) \\
\text { (without driving } \\
\text { signal) }\end{array}$} & \multirow{2}{*}{\multicolumn{4}{|c|}{$\begin{array}{c}\text { SB }(\mathrm{MHz}) \text { (with driving signal) } \\
\text { Driving signal power }\end{array}$}} \\
\hline \multirow[t]{2}{*}{$k^{21}$} & \multirow[t]{2}{*}{$k^{12}$} & & & & & \\
\hline & & & $1.44 \mathrm{~mW}$ & $2.28 \mathrm{~mW}$ & $3.61 \mathrm{~mW}$ & $5.73 \mathrm{~mW}$ \\
\hline 0.31 & 0.002 & 55 & 0 & 0 & 0 & 0 \\
\hline 0.31 & 0.10 & 43 & 3 & 4 & 5 & 7 \\
\hline 0.31 & 0.25 & 33 & 4 & 6 & 7 & 10 \\
\hline 0.05 & 0.25 & 22 & 9 & 13 & 18 & 20 \\
\hline
\end{tabular}




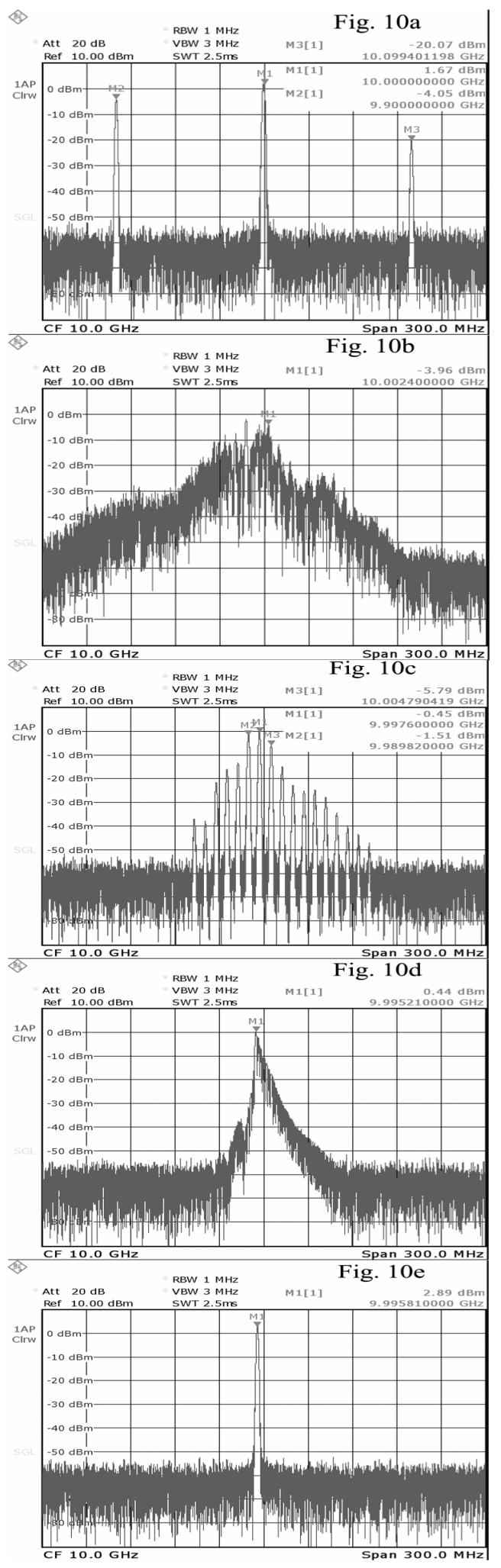

Figure 10. Spectral characteristics of DBCGO for different $f^{d}\left(P^{d}=5.73 \mathrm{~mW} ; f^{0}=10.00 \mathrm{GHz}, k^{21}=0.31, k^{12}=0.25\right)$ : (a) $f^{d}=9.9 \mathrm{GHz}$, (b) $f^{d}=9.988 \mathrm{GHz}$, (c) $f^{d}=9.990 \mathrm{GHz}$, (d) $f^{d}=9.995 \mathrm{GHz}$, (e) $f^{d}=9.996 \mathrm{GHz}$.

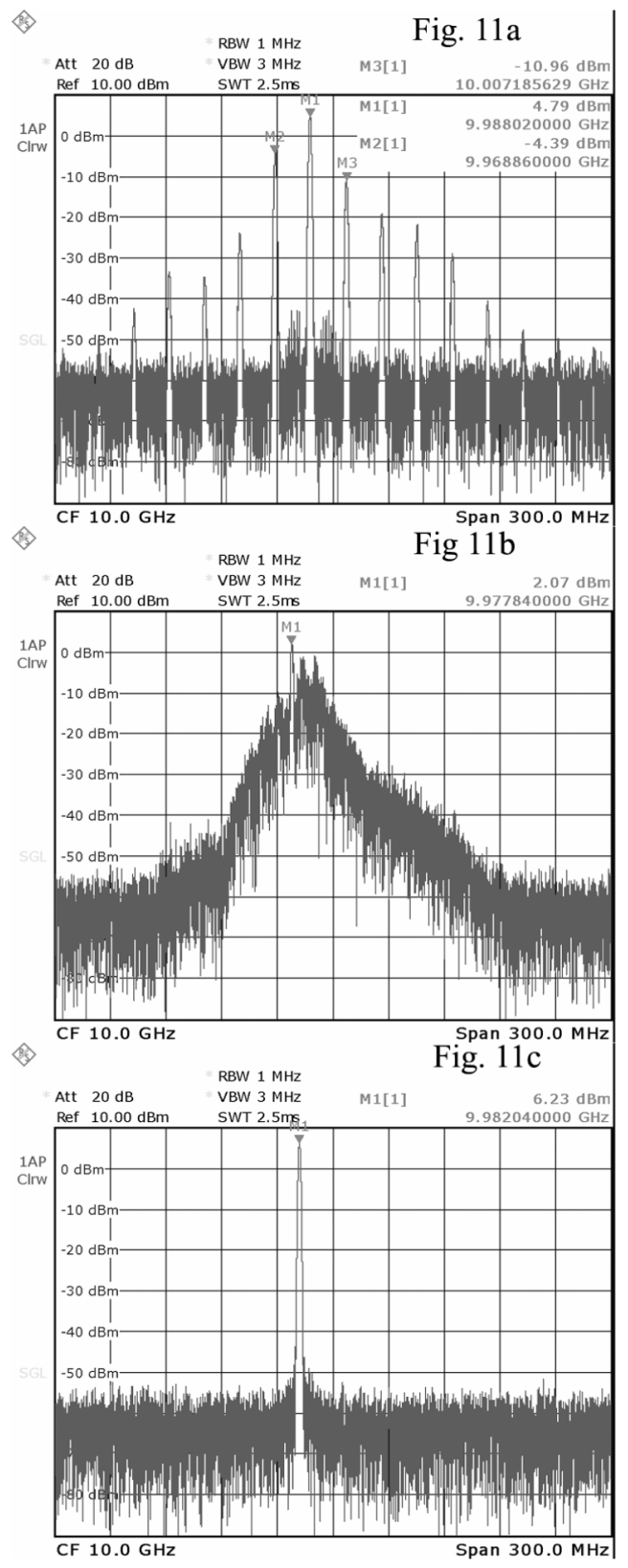

Figure 11. Spectral characteristics of DBCGO for different $f^{d}\left(P^{d}=5.73 \mathrm{~mW} ; f^{0}=9.991 \mathrm{GHz}, k^{21}=0.05, k^{12}=0.25\right)$ : (a) $f^{d}=9.969 \mathrm{GHz}$, (b) $f^{d}=9.978 \mathrm{GHz}$, (c) $f^{d}=9.982 \mathrm{GHz}$.

circuit theoretic model and physical principle of operation of the DBCGO using microwave circuit components, a set of coupled differential equations of the DBCGO system has been discussed. These equations are solved numerically using 4 th order RK method. The tuning characteristic of individual oscillator, mutual SB of the oscillators in coupled mode for a set of CFs and responses of DBCGO system have been obtained through numerical 
study. For some driving signal conditions, broad continuous spectrum as well as double-scroll type attractors have been observed. Hardware experiments done with GOs in X-band. For different set of CFs, driving signal power and frequencies, quasiperiodic, chaotic and periodic oscillations are observed. Thus one can conclude that the DBCGO system with suitable CFs and driving signal conditions (i.e. suitable set of $f^{d}$ and $P^{d}$ ) could be a potential source of chaotic signals in microwave frequency range.

\section{Acknowledgement}

Authors acknowledge partial financial assistance from DST (India) and DAE, BRNS (India) through sponsored research projects in carrying out the work.

\section{References}

1. Boi S, Couzin ID, Buono ND, Franks NR, Britton NR. Coupled oscillators and activity waves in ant colonies. Proceedings of Royal Society. 1998: 371-78.

2. Glova FA. Phase locking of optically coupled lasers. Quantum Electronics. 2003; 33(4): 283-306.

3. Stori WD et al. Dynamics of two strongly coupled Van der Pol oscillators. International Journal of Nonlinear Dynamics. 1982; 7(3):143-52.

4. Anishenkol V, Astakhov S, Vadivasova T. Phase dynamics of two coupled oscillators under external periodic force. A
Letters Journal Exploring The Frontiers of Physics. 2009; 86(3):30003(p1 - p5).

5. Uwate Y, Nishio Y. Synchronization phenomena in van der Pol oscillators coupled by a time varying resistor. International Journal of Bifurcation and Chaos. 2007; 17(10):3565-69.

6. Volos CK, Kyprianidis IM, Stouboulos IN. Experimental synchronization of two resistively coupled Duffing type circuits. Nonlinear Phenomena in Complex Systems. 2008; 11(2):187-92.

7. York RA. Nonlinear analysis of phase relationships in quasi-optical oscillator arrays. IEEE Trans Microw Theor Tech. 1993; 41(10):1799-809.

8. Georgiadis A, Collado A. Injection locked coupled oscillator arrays. IEEE Microw Wireless Compon Lett. 2007; 17(12):900-2.

9. Ram RJ, Sporer R, Blank HR, York RA. Chaotic dynamics in coupled microwave oscillators. IEEE Trans Microw Theor Tech. 2000; 48:1909-16.

10. Sarkar BC, Sarkar D, Sarkar S, Chakravorty J. Studies on the dynamics of bilaterally coupled X-band Gunn oscillators. Progress in Electromagnetic Research B. 2011; 32:149-67.

11. Hayashi C. Nonlinear Oscillations in Physical Systems. New York: Mc-Graw-Hill Book Company, (Chapter 5); 1964.

12. Sarkar BC, Koley C, Guin AK, Sarkar S. Some numerical and experimental observations on the growth of oscillations in an X-band Gunn oscillator. Progress In Electromagnetics Research B. 2012; 40:325-41. 